\title{
Green-to-blue Triplet Fusion Upconversion Sensitized by Anisotropic CdSe Nanoplatelets
}

Zachary A. VanOrman, ${ }^{1, \#}$ Alexander S. Bieber, ${ }^{1}$ Sarah Wieghold, ${ }^{1}$ Lea Nienhaus ${ }^{1, *}$

${ }^{1}$ Department of Chemistry and Biochemistry, Florida State University, Tallahassee, FL 32306, USA

*corresponding Author: $\underline{\operatorname{lnienhaus@,fsu.edu}}$

KEYWORDS: green-to-blue upconversion, CdSe nanoplatelets, transmitter ligands, triplet energy transfer, anisotropy 


\section{ABSTRACT}

Green-to-blue photon upconversion bears great potential in photocatalytic applications. However, current hybrid inorganic-organic upconversion schemes utilizing spherical CdSe nanocrystals are often limited by energetic polydispersity, low quantum yields and an additional tunneling barrier resulting from the necessity of surface-passivating inorganic shells. In this contribution, we introduce anisotropic $\mathrm{CdSe}$ nanoplatelets as triplet sensitizers. Here, quantum confinement occurs in only one direction, erasing effects stemming from energetic polydispersity. We investigate the triplet energy transfer from the CdSe nanoplatelets to the surface-bound triplet acceptor 9anthracene carboxylic acid. We further focus on the influence of nanoplatelet stacking and singlet back transfer on the observed upconversion efficiency. We obtain an upconversion quantum yield of $5.4 \%$ at a power density of $11 \mathrm{~W} / \mathrm{cm}^{2}$ using the annihilator 9,10-diphenylanthracene, and a low efficiency threshold $I_{\text {th }}$ of $237 \mathrm{~mW} / \mathrm{cm}^{2}$.

\section{TOC Graphic}

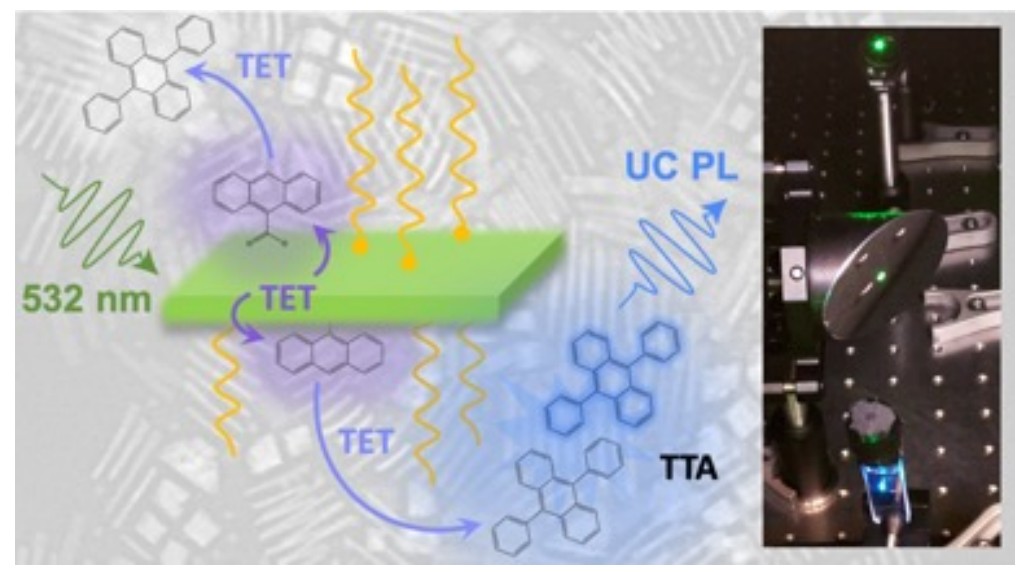


Photon upconversion (UC) describes the process of effectively shortening the wavelength of emission upon optical irradiation. In order to adhere to energy conservation laws, this process involves the combination of a minimum of two low-energy photons to create one high-energy photon. In particular, in the triplet fusion (TF) process investigated here, this 'anti-Stokes'-type emission is the result of a spin-allowed triplet-triplet annihilation (TTA) process: Two low energy spin-triplet states are combined in the annihilator molecule in a spin-allowed process to form a higher lying singlet state, as well as a ground state singlet.

Depending on the wavelength created by the UC process, there are several different applications: (i) near-infrared (NIR)-to-visible UC bears promise in biomedical applications, ${ }^{1,2}$ biological imaging, ${ }^{3}$ and infrared sensitization of silicon solar cells ${ }^{4,5}$ or in the fabrication of low-cost infrared sensing cameras. ${ }^{6}$ (ii) Green-to-blue or blue-to-ultraviolet UC on the other hand, bears promise in photochemical applications by transiently creating the high energies required for catalysis, thus avoiding photobleaching issues. ${ }^{7-11}$

As the UC process relies on spin-triplet states, which are deemed as "spin-forbidden" due to spin selection rules and hence, cannot be efficiently populated by direct optical excitation, we rely on triplet sensitizers for the creation of triplet states by a triplet energy transfer (TET) process following either the Marcus ${ }^{12}$ or Dexter ${ }^{13}$ theory. Reports by Castellano and others indicate that metal-organic complexes with strong spin-orbit coupling resulting from inclusion of heavy metal atoms can act as efficient triplet sensitizers for TTA-UC., ${ }^{71-22}$ While high UC efficiencies exceeding $20 \%$ have been reported in such systems, ${ }^{23,24}$ one of the main limitations is the large energy gap between the singlet and the triplet state in these metal-organic complexes. As a result, over $300 \mathrm{meV}$ energy losses can occur during the intersystem crossing (ISC) process required to create the triplet state. ${ }^{16,20}$ This large energy loss effectively limits the energy gain possible during 
the UC process. Therefore, other material classes have been investigated as triplet sensitizers. In particular, inorganic nanocrystals such as quantum dots (QDs) have shown great promise due to strong spin-orbit coupling resulting from the abundance of heavy metals in these particles and wavefunctions that do not exhibit distinct singlet or triplet character but rather a superposition of both. ${ }^{25}$ Therefore, QDs can act as efficient triplet sensitizers with minimal energy losses with reports of UC efficiencies exceeding $16 \% .{ }^{25-29}$

However, QDs often suffer from low quantum yields (QYs), necessitating inorganic core-shell structures $^{30,31}$ and additionally require long passivating ligands for surface passivation and colloidal stability. These long ligands hinder efficient TET due to its exponential distance dependence. To overcome this hurdle, bound transmitter ligands have been employed to efficiently funnel the triplet exciton through the organic shell to the freely diffusing annihilator molecules..$^{32,33}$ Here, we present a new class of triplet sensitizers: two-dimensional (2D) inorganic nanoplatelets (NPLs). ${ }^{34-42}$ NPLs have several properties which may prove advantageous over spherical zerodimensional (0D) QDs: (i) high intrinsic photoluminescence (PL) QYs which circumvent the requirement of an inorganic shell to passivate surface trap states, (ii) quantum-confinement in only one direction: the lateral dimensions do not affect the energetics of the NPL, and only the thickness of the platelets is important. Hence, only homogeneous broadening of the emission is observed, and no inhomogeneous broadening resulting from size polydispersity. (iii) Giant oscillator strengths which result in large absorption cross-sections and fast radiative lifetimes. ${ }^{35}$

While the TET mechanism is fairly well studied for spherical QDs, there is no precedence for how TET occurs when using anisotropic NPLs. Since the underlying properties of NPLs have not been studied as extensively as for standard spherical 0D QDs, the question arises what the nature of the 'triplet' state in such a 2D NPL is. Is there a preferred transition in the NPL that undergoes TET, 
or a directionality or polarization that is required? To begin to answer these questions, we integrate the NPLs into our solution-based TTA-UC system.

We take advantage of the unidirectional properties of transmitter ligands to enhance the probability of TET through the organic ligand shell. We combine such a transmitter ligand 9-anthracenecarboxylic acid (ACA) with the anisotropic 2D CdSe NPLs and demonstrate green-to-blue UC. Briefly, the CdSe NPLs (5.5 monolayer thickness, excitonic feature at $545 \mathrm{~nm})^{43,44}$ act as the triplet sensitizer, ACA is the transmitter ligand, and TTA-UC occurs in the established annihilator molecule 9,10-diphenylanthracene (DPA). For direct comparison of the results of the 2D NPLs with the established 0D QD triplet sensitizers, we subject the QD control sample to the same conditions as the NPLs, which is detailed in the Supporting Information.
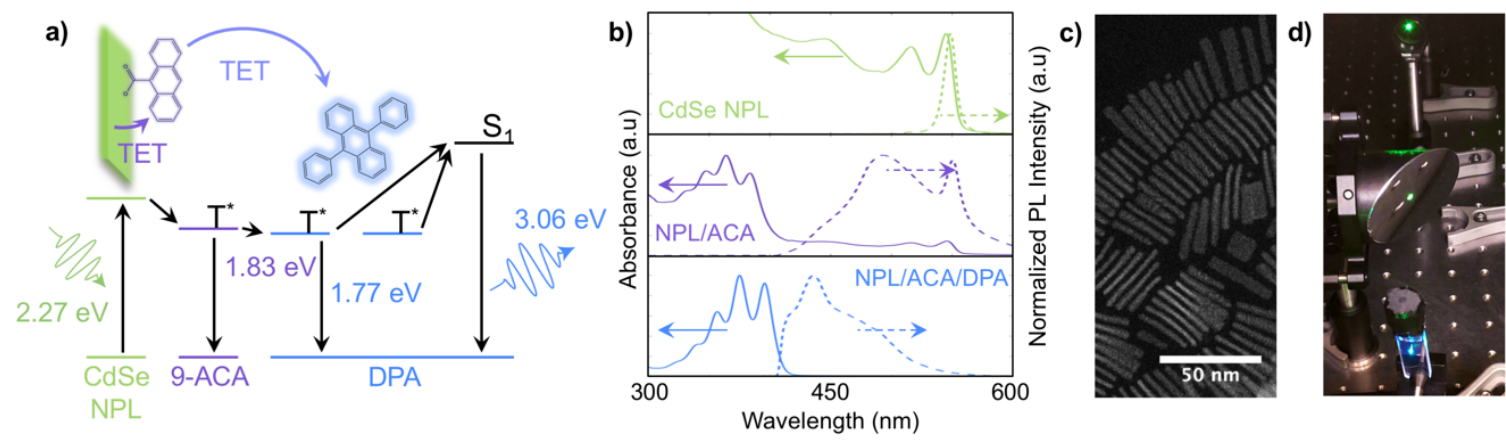

Figure 1: a) Schematic of the relative energies of the NPL exciton (green), triplet excited state of the 9-ACA transmitter ligand (purple), triplet excited state of the DPA triplet acceptor, and DPA singlet excited state (blue) and the energy transfer cascade occurring. b) Normalized absorbance (solid line) and PL (dashed line) of the 5.5 monolayer CdSe NPL (green), NPL/ACA (purple), and NPL/ACA/DPA (blue). c) Representative STEM image of the synthesized CdSe NPLs. d) Photograph of the observed upconverted emission ( 430 nm) from the NPL/ACA/DPA solution upon 532 nm excitation.

Figure 1a shows a schematic of the relative energies of the NPL exciton, the triplet excited state of ACA and DPA, as well as the singlet energy of DPA resulting in an overall energy gain of $\sim 0.6$ $\mathrm{eV}$ during the UC process. Representative absorption (solid lines) and normalized emission spectra (405 nm excitation, dashed line) of the CdSe NPLs (green), NPLs with the transmitter ligand ACA 
added (NPL/ACA, purple), as well as the UC solution composed of NPL/ACA and DPA (blue) are shown in Figure 1b. In presence of ACA, we observe a slight red-shift of the NPL absorption onset and the peak of the emission spectra, indicating a change in the dielectric environment due to coupling of the ligands (SI Figure S1). ${ }^{45,46}$ A representative STEM image of the 2D NPLs is shown in Figure 1c, highlighting the 2D structure of the NPLs. Compare SI Figure S2 for the corresponding QD/ACA/DPA energy alignment and optical properties. A photograph of the 'antiStokes' emission obtained by the UC process is shown in Figure 1d: under $532 \mathrm{~nm}$ excitation, we obtain $\sim 430 \mathrm{~nm}$ emission.

To investigate the TET process from CdSe NPLs to the ACA transmitter ligand in more detail, we first investigate the relative CdSe NPL quenching behavior as a function of the transmitter ligand concentration by exploring five different concentrations of ACA. Figure 2a shows the steady-state quenching of the NPL solution with an increasing amount of ACA. As expected, with an increasing ACA concentration, the NPL photoluminescence at $546 \mathrm{~nm}$ is more quenched.

Time-resolved PL spectroscopy yields insight on the underlying dynamics of the TET process. Figure $2 b$ shows the PL decay dynamics of the CdSe NPLs in solution with varying amounts of ACA. The obtained lifetimes agree with the steady-state PL results, indicating a higher amount of quenching with an increasing ratio of the ACA transmitter ligand (compare SI Figure S3 for the QD steady-state quenching and decay dynamics).

For comparison, we also investigate the quenching behavior when DPA is added to either the NPL/ACA system as well as to the bare NPL solution to confirm our hypothesis that the transmitter ligands are required to efficiently extract the exciton from the NPLs. Since the triplet extraction occurs predominately through the transmitter ligand, little additional quenching is expected by the presence of DPA (compare SI Figure S4). This observation is further confirmed by a control 
experiment, in which the NPLs or QDs are simply mixed with DPA (SI Figure S4). Neither quenching of the PL lifetime, nor visible blue emission caused by UC can be observed, indicating that the transmitter ligands are indeed required to efficiently extract the exciton from the NPLs to the triplet state of DPA.

a)

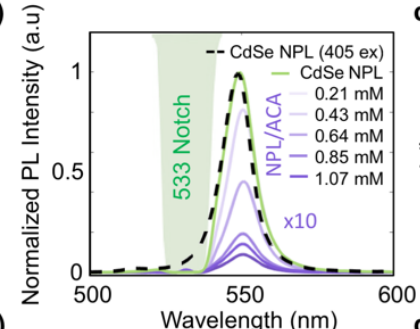

b)

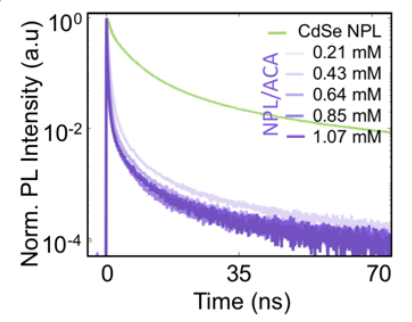

c)
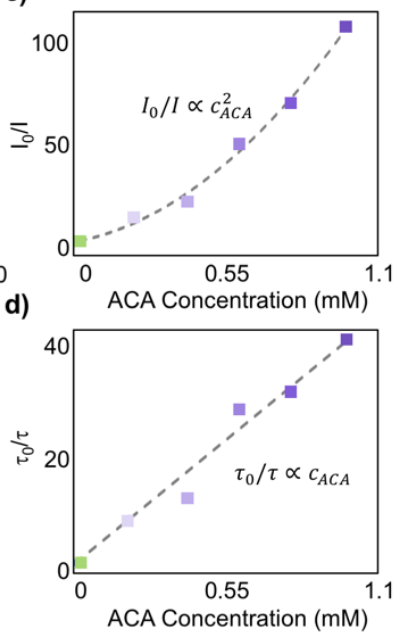

Figure 2: Quenching properties of the NPL with various amounts of ACA transmitter ligand. a) Steady-state PL quenching of the CdSe NPL emission upon the addition of different concentrations of ACA (purple). For clarity, the quenched PL spectra have been multiplied by a factor of 10. CdSe NPL emission under $405 \mathrm{~nm}$ (black dotted line) is also added, as some emission is blocked by a 533 notch filter highlighted in green. b) Time-resolved PL quenching of the CdSe NPL emission.. c) Stern-Volmer plot detailing the ratio of the intensities as a function of the ACA concentration. d) Stern-Volmer plot utilizing the ratio of the decay lifetime $\tau$ as a function of the ACA concentration.

To further investigate the quenching dynamics in more detail, we plot both the NPL steady-state emission intensity $\mathrm{I}_{0} / \mathrm{I}$ (Figure $2 \mathrm{c}$ ) and the lifetime ratio $\tau_{0} / \tau$ (Figure $2 \mathrm{~d}$ ) as a function of the ACA quencher concentration for the NPL system in a Stern-Volmer plot. The extracted lifetimes and relative quenching percentages are summarized in Table 1. 
Table 1. Final ACA concentration during ligand exchange, relative quenching and lifetimes of the NPL/ACA and $Q D / A C A$ coupled system. The lifetimes marked with * are similar the instrument response function: $\tau_{I R F} \approx 0.1 \mathrm{~ns}$ and may have a large error.

\begin{tabular}{|r|r|r|r|r|}
\hline ACA concentration & $\begin{array}{c}\text { NPL Lifetime } \\
\left(\tau_{N P L}\right)\end{array}$ & \multicolumn{1}{c|}{$\begin{array}{c}\text { Relative } \\
\text { quenching }\end{array}$} & $\begin{array}{c}\text { QD Lifetime } \\
\left(\tau_{N P L}\right)\end{array}$ & $\begin{array}{c}\text { Relative } \\
\text { quenching }\end{array}$ \\
\hline- & $2.9 \mathrm{~ns}$ & $0 \%$ & $14.5 \mathrm{~ns}$ & $0 \%$ \\
\hline $0.21 \mathrm{mM}$ & $0.32 \mathrm{~ns}$ & $89 \%$ & $0.54 \mathrm{~ns}$ & $96 \%$ \\
\hline $0.43 \mathrm{mM}$ & $0.23 \mathrm{~ns}$ & $92 \%$ & $0.36 \mathrm{~ns}$ & $97 \%$ \\
\hline $0.64 \mathrm{mM}$ & $0.10 \mathrm{~ns}^{*}$ & $96 \%$ & $0.32 \mathrm{~ns}$ & $98 \%$ \\
\hline $0.85 \mathrm{mM}$ & $0.09 \mathrm{~ns}^{*}$ & $97 \%$ & $0.27 \mathrm{~ns}$ & $98 \%$ \\
\hline $1.07 \mathrm{mM}$ & $0.07 \mathrm{~ns}^{*}$ & $98 \%$ & $0.21 \mathrm{~ns}$ & $99 \%$ \\
\hline
\end{tabular}

The quadratic increase of the intensity quenching and the linear increase of the lifetime indicate a combination of both collisional and static quenching for both the NPLs and the QDs (compare SI Figure S3). From the lifetime quenching, we extract a bimolecular quenching constant of $1.3 \mathrm{x}$ $10^{13} \mathrm{M}^{-1} \mathrm{~s}^{-1}$. In comparison, we extract a bimolecular quenching constant of $2.8 \times 10^{12} \mathrm{M}^{-1} \mathrm{~s}^{-1}$ for the QD.

These observations so far have indicated that NPLs can be used as efficient triplet sensitizers. However, to gain more insight into the underlying mechanism resulting in UC, we investigate the properties of the upconverted emission. In Figure 3a, we show a representative emission spectrum of our NPL UC system under $532 \mathrm{~nm}$ excitation. The PL spectrum obtained when directly exciting DPA at $405 \mathrm{~nm}$ is highlighted as dashed line for comparison. Clearly visible in the spectral region from $400-500 \mathrm{~nm}$ is the blue emission stemming from the UC process. 
a)

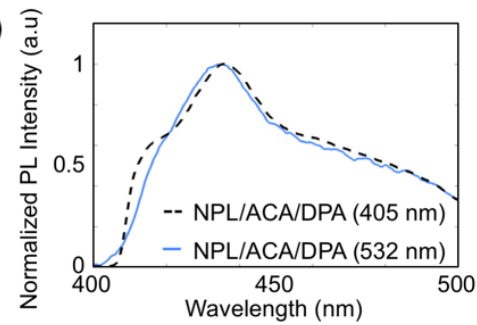

b)
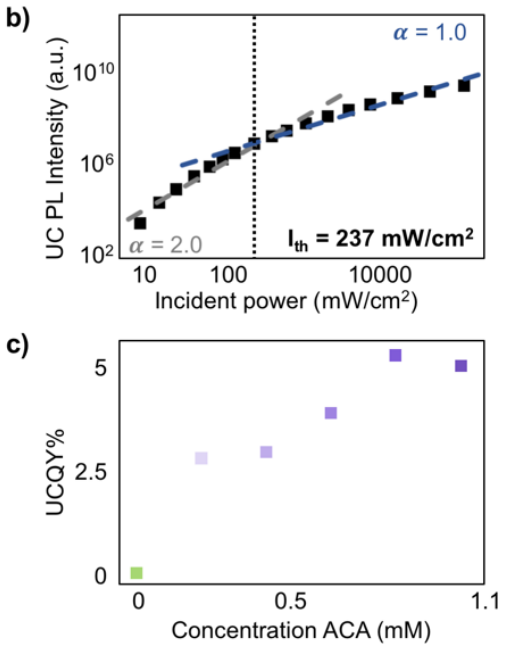

Figure 3: a) DPA emission under 405 excitation (black dotted line) and UC emission under 532 nm excitation (blue). b) Logarithmic plot of the UC PL intensity versus different laser fluences, resulting in a characteristic 2 to 1 change in the slope expected in systems with TTA based UC. c) The upconversion QY plotted as a function of the ACA concentration.

UC occurring through the TTA process follows a unique power dependency. ${ }^{14,18,19}$ At low excitation powers below the intensity threshold $I_{\text {th }}$, a slope of $\alpha=2$ can be observed, indicative of a bimolecular process. In this regime, TTA is inefficient and quasi-first order triplet recombination pathways can be observed. This is a result of pathways including phosphorescence, non-radiative relaxation and other quenching processes to the ground state. Above the $I_{\text {th }}$, TTA becomes efficient and is the predominant relaxation pathway, resulting in the creation of high-energy singlets. As a result, the $I_{\text {th }}$ yields insight on how efficient the UC process is at a given incident power (eq. 1):

$$
I_{t h}=\frac{\left(k_{A}^{T}\right)^{2}}{\Phi_{T E T} \alpha(E) \gamma_{T T}}
$$

where $k_{A}^{T}$ is the nonradiative triplet decay rate, $\Phi_{T E T}$ is the efficiency of TET, $\alpha(E)$ the absorption coefficient, and $\gamma_{T T}$ the second-order rate constant characterizing the TTA process. As shown in 
Figure $3 \mathrm{~b}$, we obtain a slope change from $\alpha=2$ to $\alpha=1$ at the intensity threshold $I_{\text {th }}=237 \mathrm{~mW} / \mathrm{cm}^{2}$, which is lower than the value of $574 \mathrm{~mW} / \mathrm{cm}^{2}$ obtained for the QD/ACA/DPA system at a similar optical density (compare SI Figure S5), despite the nearly two orders of magnitude lower concentration of the NPL sample.

The UC efficiency $\Phi_{U C}$ (eq. 2) is defined as the product of intersystem crossing (ISC) $\Phi_{I S C}$, energy transfer from the sensitizer to the annihilator $\Phi_{T E T}$ and the TTA efficiency $\Phi_{T T A}$, normalized by the annihilator QY $\Phi_{a n n}: 47-49$

$$
\Phi_{U C}=f \Phi_{I S C} \Phi_{T E T} \Phi_{T T A} \Phi_{a n n}
$$

The factor $f$ is a statistical factor which gives insight on the probability of creating a singlet state from two annihilator singlet states. We calculate the relative UC efficiency for each transmitter ligand concentration (Figure $3 \mathrm{c}$ ) and achieve the highest value of $5.4 \%$ for $0.85 \mathrm{mM} \mathrm{ACA}{ }^{49}$ In comparison, we obtain a $16 \%$ efficiency for the ACA-coupled QDs, which is comparable with values reported for spherical CdSe by Tang and co-workers. ${ }^{29}$ Table 2 shows the calculated UC efficiencies for both the NPL and QD-based UC systems for all ACA concentrations. For the efficiency calculations we refer to the Supporting Information and Figure S6 for more details.

Table 2: UCQYS of the NPLs and QDS as a function of the added ACA transmitter ligand concentration.

\begin{tabular}{|r|r|r|}
\hline ACA Concentration (mM): & NPL UCQY (\%) & QD UCQY (\%) \\
\hline $0 \mathrm{mM}$ & N/A & $0.8 \%$ \\
\hline $0.21 \mathrm{mM}$ & $2.7 \%$ & $12 \%$ \\
\hline $0.43 \mathrm{mM}$ & $2.4 \%$ & $14 \%$ \\
\hline $0.64 \mathrm{mM}$ & $3.8 \%$ & $13 \%$ \\
\hline $0.85 \mathrm{mM}$ & $5.4 \%$ & $16 \%$ \\
\hline $1.07 \mathrm{mM}$ & $5.0 \%$ & $16 \%$ \\
\hline
\end{tabular}

By having a closer look into these values, obtaining a lower $I_{\text {th }}$ value, yet a lower UC efficiency is counterintuitive as this indicates that the TTA process becomes more efficient at a lower power, 
yet is less efficient overall. The variables in eq. $2 \Phi_{T T A}, \Phi_{a n n}$ and $f$ are expected to be independent of the sensitizer. That leaves $\Phi_{I S C}$ and $\Phi_{T E T}$ to be responsible for this unexpected discrepancy. To discuss their possible roles in the UC process, we investigate the underlying meaning of these terms. ISC describes the process of transitioning between two states with different spin multiplicity. However, electron spin is not a good quantum number in these materials, rather the total angular momentum is the relevant quantum number. Instead, the exchange energy between bright and dark exciton states with singlet and triplet character can give insight on whether a material can be used as a triplet sensitizer. As shown by Shornikova et al., ${ }^{50}$ who investigated the excitonic fine structure of CdSe NPLs, the bright-dark exchange energy for 2D NPLs is smaller than the available thermal energy $k T$, indicating that $\Phi_{I S C}$ is unlikely to be an underlying cause of the lower UC efficiency.

The triplet transfer efficiency is defined as the fraction of excitons created in the sensitizer which are quenched via TET to the triplet acceptor. As shown previously in Table 1, both steady-state emission and the lifetime of the NPL/ACA system is highly quenched, indicating an interaction between the NPL and ACA, which can be approximated to a near-unity $\Phi_{T E T}$ to the transmitter ligand, as observed previously in QDs. This is further supported by the fact that the $I_{\text {th }}$ is also dependent on $\Phi_{T E T}$, and a low TET efficiency would be reflected here.

However, since these parameters are usually used as a figure of merit to describe the UC efficiency, we would like to highlight additional loss mechanisms which are yet not mentioned. The first one to highlight is back transfer of emissive singlets created in DPA to the sensitizer via Förster resonance energy transfer (FRET). As the UC efficiency is not unity and can only create one singlet for two triplet states created, a recycling of the upconverted emission via FRET would result in an overall decrease of the emitted intensity however, should not affect the $I_{\text {th }}$ value. Further, as 
investigated by Meinardi and co-workers, $I_{\text {th }}$ values can be lowered by using donors with high absorption cross sections or by increasing the donor concentration. However, we note that an increase in the sensitizer absorption likely also results in increased singlet back transfer for our broadband absorbing sensitizers. ${ }^{18}$

To investigate if the effect of singlet back transfer via FRET is the underlying cause of the discrepancy of our UC efficiency measurement, we vary the NPL sensitizer concentration and study the impact of the sensitizer concentration on the $I_{t h}$ value and UC efficiency (compare Figure S7 for the concentration dependence of the QD-based UC system).

Figure 4a highlights the change in the $I_{\text {th }}$ value as a function of the relative concentration of the sensitizer in comparison to the previously used NPL concentration $c_{0}=2.6 \times 10^{-8} \mathrm{~mol} / \mathrm{L}$. The corresponding relative UC efficiencies for each concentration are plotted in Figure 4b. As expected, a dilution below the previously optimized sensitizer concentration increases the $I_{\text {th }}$ value and reduces the obtained UC efficiency. ${ }^{18}$ On the other hand, an increase in the sensitizer concentration yields the expected decrease in the $I_{t h}$ value but no increase in the UC efficiency. Rather, a decrease in the UC efficiency compared to $c_{0}$ is observed. This indicates that the UC process becomes efficient at lower incident light densities for concentrations larger than $c_{0}$, yet less light is emitted overall. Therefore, care must be taken not to confuse the $I_{\text {th }}$ value with the overall observed brightness of the UC process, as these may not correlate.

In the following we will elucidate how both effects can be decoupled and explained by various assumptions as well as provide a hypothesis for the obtained counterintuitive results of the $I_{\text {th }}$ value and the UC efficiency. First, to shine light onto the reduction in the UC brightness, we consider an increase in the FRET efficiency of back-transfer. Here, the giant oscillator strength of the NPLs, which we estimate to be about 100-fold larger than that of the 0D QDs, results in an increased 
dipole-dipole coupling between the NPL and the annihilator DPA, thus an increase in the FRET radius. ${ }^{51}$ Further, taking into consideration that 2D NPLs have a non-negligible lateral extension, the underlying assumption of point dipoles may also not hold. In the case of $0 \mathrm{D} \rightarrow 2 \mathrm{D}$ energy transfer, the commonly accepted $1 / R^{6}$ distance dependence of the FRET efficiency reverts to a $1 / R^{4}$ dependency, thus, further extending the distance over which FRET can occur. ${ }^{52}$ In addition, as we increase the sensitizer concentration, the distance between the donor (DPA) and the acceptor (NPL) decreases. This indicates that FRET may be more predominant for the NPLs than for the QD-based system. Indeed, we do not observe the same strong effect for the QDs despite these being at the same optical density, and therefore at a higher molar concentration or a lower donoracceptor distance. Following these assumptions, we estimate the relative FRET transfer efficiency as a function of the relative distance $R / R_{0}$ of the QDs (gray), NPLs (blue) considered as a point dipole, and NPLs considered as a 2D material (green) as shown in Figure $4 c{ }^{52}$ Based on the distance dependence shown, we conclude that the decrease in the observed UC efficiency is not consistent with the effect expected when linearly increasing the NPL (QD) concentration by an order of magnitude, thus decreasing the NPL-NPL spacing roughly two-fold.
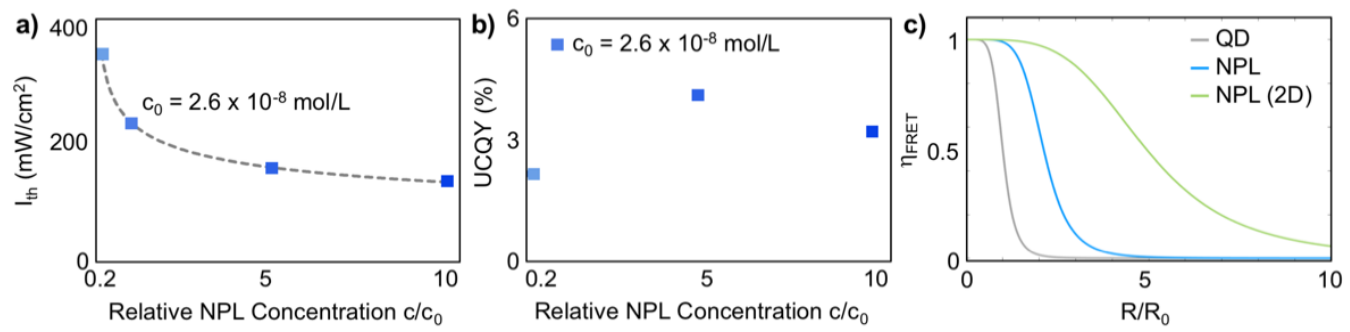

Figure 4: a) $I_{t h}$ as a function of the NPL concentration relative to the previously used NPL concentration $\mathrm{c}_{0}=2.6 \times 10^{-}$ ${ }^{8} \mathrm{~mol} / \mathrm{L}$ ) with fixed concentrations of ACA and DPA. The dashed line serves as a guide to the eye. b) UC QY at the same concentrations of a). c) Simulated FRET efficiencies as a function of the relative FRET radius for quantum dots (gray), NPLs as point dipoles (blue), and NPLs in two dimensions (green). 
Therefore, we discuss an additional effect that occurs selectively in the NPL system and its effect on the UC efficiency: NPL stacking. To debate how NPL stacking influences the UC process, we investigate the underlying processes that take place. For TTA-UC to occur in our tri-component TTA-UC system, two criteria must be met: (i) the triplet must be funneled through the ligand shell by ACA to DPA molecules freely diffusing in solution and, (ii) two DPA molecules excited into the correct spin-opposite triplet state must collide. Since the latter is independent of the sensitizer, we will focus on the first assumption in the following.

Due to Van der Waals interactions between NPLs, aggregates readily form which may hinder the required TET from the transmitter ligand to the annihilator DPA. ${ }^{53,54}$ Here, an increase in the NPL concentration would lead to an increase in the amount of stacking, resulting in a similar concentration or separation of 'particles' in the solution, just of varying aggregate sizes. As a result, the amount of back transfer would not be influenced to the expected extent. We conclude that back transfer is not likely to be the main culprit of the low observed UC QYs.

However, NPL stacking also may cause other underlying effects, which have not been considered yet. As the schematic in Figure 5a shows, buried ACA ligands may indeed quench the NPL, yet cannot undergo TET to the freely diffusing DPA. As a result, the quenching of the NPL may not be a good indicator for the efficiency of TET from the NPL to the DPA, and therefore for the number of triplets created in the annihilator.

This hypothesis is further supported by some unusual effects observed in the NPL-based UC power dependence, in particular at high NPL concentrations, which results in NPL stacking. As mentioned previously, TTA shows a unique power dependence with a slope change from $\alpha=2$ to $\alpha=1$. However, we observe two odd effects in the UC intensity when using a high NPL concentration: i) a slope above $\alpha=2$ for very low powers, and ii) a correlated photobrightening 
of the observed upconverted emission under constant excitation power, as shown in Figure $5 \mathrm{~b}$. While slopes above $\alpha=2$ can be explained if the triplet sensitization occurs through a nonexcitonic process, ${ }^{55,56}$ or the excitons are created by a bimolecular process such as two photon absorption, ${ }^{57}$ this is not the case here. As Figure S8 shows, the NPL emission power dependency is linear for all concentrations, indicating the expected excitonic behavior for this material type. The increased slope above $\alpha=2$ can also be understood as the $\mathrm{UC}$ emission being lower than expected. This is consistent with a quenching to non-active ACA transmitter ligands, which cannot transfer their triplet exciton to the freely diffusing DPA molecules due to steric hinderance by stacking.

Further, the photobrightening of the UC at low powers (green and gray insets in Figure $5 b$ ) suggests that more TTA-active triplets are created over time under continuous illumination. This is also in agreement with a quenching process to inactive transmitter ligands. The inaccessible TET-inactive transmitter ligands can act as non-radiative 'trap-states', which are filled over time. Due to the longevity of the spin-triplet states, these states will stay filled for several microseconds until they non-radiatively decay, while the accessible transmitter ligands are rapidly quenched to DPA. As the inactive transmitter ligand triplet states are filled, the NPLs begin to predominately funnel their energy into the active transmitter ligands which result in observable TTA. Since the absorbed photons are now actively funneled into predominately TTA-active ligands, the UC emission photobrightens until an equilibrium is achieved between the population of the active and inactive transmitter ligands. At higher excitation powers, the inactive transmitter ligand states are filled more rapidly, and the equilibrium is shifted to the active transmitter ligands, resulting in a reduction of the effect on the UC emission. 
Lastly, we investigate the effect of NPL stacking prior to ligand exchange with ACA, as depicted in the schematic in Figure 5c. For this we synthesize 'pre-stacked' NPLs using hydrated cadmium acetate precursors, which is known to change the aspect ratio of the NPLs, ${ }^{58}$ and therefore also leads to a difference in the stacking behavior. As shown by Table S1 and Figure S9, stacked NPLs can easily be identified by a more quenched lifetime, due to rapid FRET between the stacked NPLs. ${ }^{53,54}$ In Figure 5d, we show the PL emission decay dynamics for pre-stacked NPLs subjected to the same ligand exchange procedure as the NPLs in Figure 2b. Based on the concentrationdependent steady-state quenching (Figure 5e and Figure S10), and the time-resolved decay dynamics (Figure $5 \mathrm{f}$ and Figure S11), we observe that the pre-stacked NPLs are not as quenched by the addition of ACA, and that the quenching intensity and lifetime saturate despite an increase of the ACA concentration. A plateau in the Stern-Volmer plot (gray shaded region in Figures 5ef) suggests that there is a sub-population of NPLs which is not interacting with the ACA transmitter ligand likely due to the inaccessibility of the transmitter ligands and therefore, are not quenched. In the elongated stacks of NPLs, the binding sites of the inner NPLs may be sterically hindered, resulting in a reduced fraction of ACA binding. 

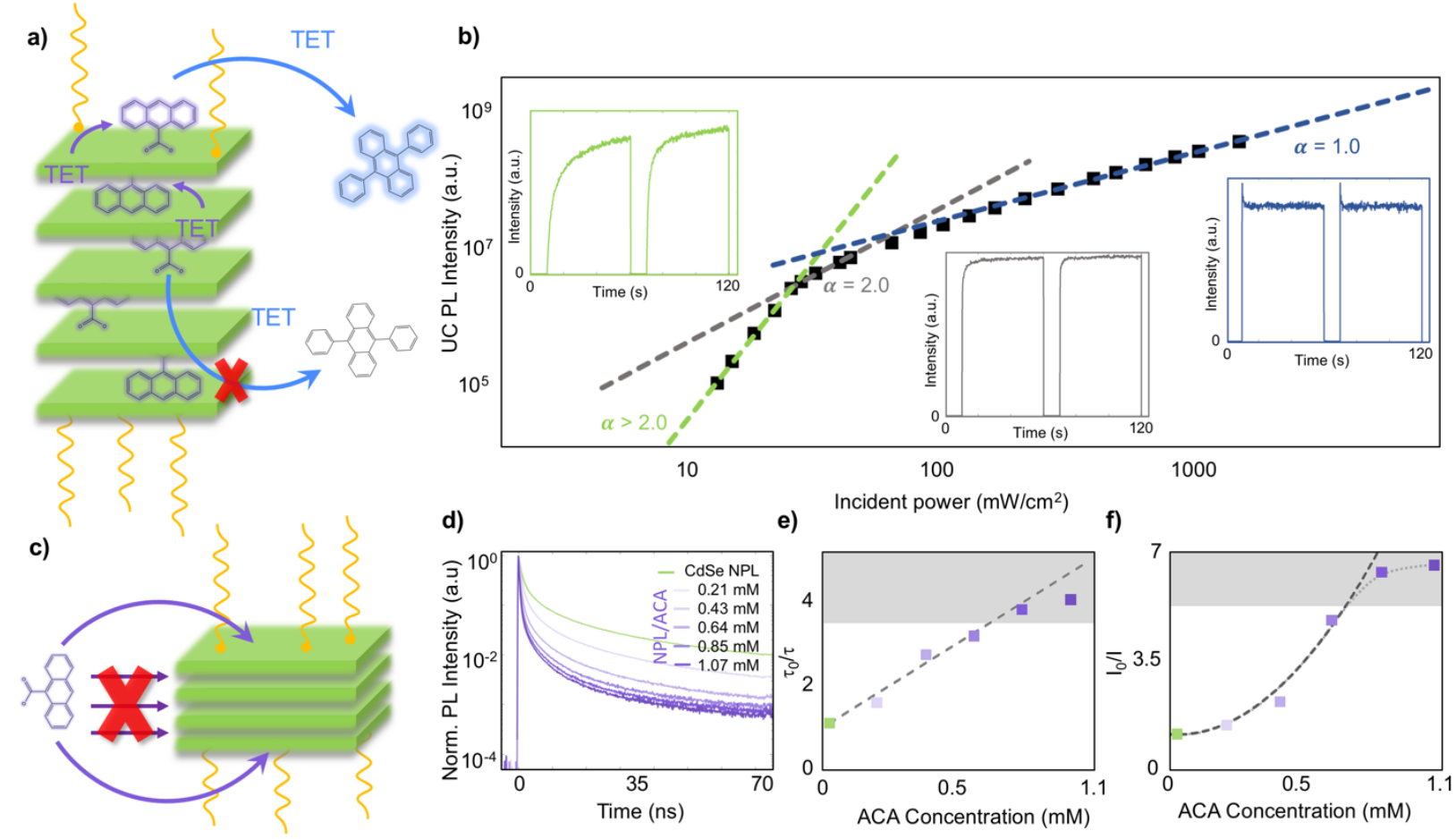

Figure 5: a) Schematic showing triplet energy transfer (TET) with stacked NPLs that have already undergone ligand exchange. While TET can occur to the ACA transmitter ligand, steric hinderance from the stacked NPLs disallows TET from buried ACA ligands to DPA. b) Plot of the UC PL intensity at different laser fluences of a sample with a high NPL concentration. There are three slope regions: a slope greater than 2 (green), where a high degree of photobrightening over time occurs (green inset), a slope of 2, (gray) where a lesser degree of photobrightening occurs (gray inset), and a slope of 1 (blue), where no photobrightening over time occurs (blue inset). c) Schematic detailing ligand exchange in prestacked NPLs. When NPLs stack, they disallow ligand exchange to occur at sites buried in the NPL stacks. d) Time-resolved PL decay in pre-stacked NPLs. e) Stern-Volmer plot utilizing the ratio of NPL lifetimes against the ACA concentration $\mathrm{f}$ ) Stern-Volmer plot examining the ratio of steady-state intensities of the NPL emission at different ACA concentrations. A quadratic fit is shown for the first four points (dashed gray). The dotted gray line highlights the plateauing of the intensity quenching. The gray shaded region highlights the region in which the SternVolmer plots deviate from the expected.

In conclusion, we have been able to show that the 2D CdSe NPLs show a lower UC threshold than comparable CdSe QDs at the same optical density. This implies that the NPLs indeed may exhibit superior properties with respect to triplet sensitization than the previously utilized spherical 0D QDs. However, the large oscillator strength of the NPLs results in strong back transfer of the UC emission and can reduce the observed UC efficiency. Stacking of the NPLs post-ligand exchange results in TET-inactive transmitter ligands, which result in quenching of the NPL emission, yet no 
TTA-active triplet states on DPA. Furthermore, pre-stacked NPLs are sterically hindered, and therefore, cannot be fully quenched by added transmitter ligands.

This study shows the promise CdSe NPLs have as triplet sensitizers, and also the current drawbacks. Engineering the NPL system to reduce stacking promises to alleviate many of these effects, and show further advancement in NPL-based TTA-UC. In addition, investigating the exact mechanism of TET, and the underlying states involved are of future interest to fully understand how the coupling occurs between the anisotropic 2D material and the transmitter ligand.

\section{MATERIALS AND METHODS}

Materials: Sodium myristate, cadmium nitrate tetrahydrate, selenium, octadecene (ODE), oleic acid (OA), chlorobenzene, hexanes, methanol, toluene, and ethanol were purchased from Millipore Sigma. 9-anthracene-carboxylic acid (ACA) and 9,10-diphenylanthracene (DPA) were purchased from TCI Chemicals. All chemicals were used as received.

$\mathrm{Cd}(\mathrm{myr})_{2}$ : Cadmium myristate was prepared according to Ref. [59]. $5 \mathrm{~g}$ of sodium myristate and $3 \mathrm{~g}$ of cadmium nitrate tetrahydrate were dissolved in 250 and $500 \mathrm{~mL}$ of methanol, respectively. The cadmium containing solution was then added dropwise to the dissolved sodium myristate solution and stirred at room temperature for $2 \mathrm{~h}$. The synthesized $\mathrm{Cd}(\mathrm{myr})_{2}$ was filtered, washed three times with methanol and dried under vacuum.

CdSe Nanoplatelet (NPL) synthesis: CdSe NPLs were synthesized according to Ref. [60] with modifications. Briefly, $193 \mathrm{mg}$ of $\mathrm{Cd}(\mathrm{myr})_{2}$ were added to $15 \mathrm{~mL}$ of ODE and degassed under vacuum at $120^{\circ} \mathrm{C}$ for $1 \mathrm{~h}$. The solution was then heated to $260^{\circ} \mathrm{C}$ under constant flow of $\mathrm{N}_{2}$ gas. $21 \mathrm{mg}$ of Se in $1 \mathrm{~mL}$ of ODE was injected to the above solution. After 45 seconds, $162 \mathrm{mg}$ of $\mathrm{Cd}(\mathrm{Ac})_{2}$ was injected. The solution was then kept at $260^{\circ} \mathrm{C}$ for $9 \mathrm{~min}$. Afterwards, the solution 
was cooled, and at $150^{\circ} \mathrm{C}, 750 \mu \mathrm{L}$ of OA was injected. The NPL solution was then transferred to a glovebox consisting of an $\mathrm{N}_{2}$ atmosphere $\left(<0.1 \mathrm{ppm} \mathrm{O}_{2}\right)$. The NPL solution was purified through centrifugation at $6000 \mathrm{rpm}$ for 15 minutes. The NPLs were then dispersed in $1 \mathrm{~mL}$ of hexanes. ACA ligand exchange was performed by adding varying amounts of a saturated solution of ACA (3.08 mM) in chlorobenzene into the NPL solution and then stirred for one hour in an air-free $\mathrm{N}_{2}$ atmosphere.

CdSe Quantum Dot (QD) synthesis: CdSe QDs were synthesized in a similar manner with a few modifications, omitting the final injection step in the NPL synthesis. $192 \mathrm{mg}$ of $\mathrm{Cd}(\mathrm{myr})_{2}$ were added to $15 \mathrm{~mL}$ of $\mathrm{ODE}$ and degassed under vacuum at $120^{\circ} \mathrm{C}$ for $1 \mathrm{~h}$. The solution was then heated to $240{ }^{\circ} \mathrm{C}$ under constant flow of $\mathrm{N}_{2}$ gas. $18 \mathrm{mg}$ of Se in $1 \mathrm{~mL}$ of ODE was then injected to the above solution. The temperature was maintained for $\sim 3$ minutes, and the solution was allowed to cool. At $\sim 150{ }^{\circ} \mathrm{C}, 500 \mu \mathrm{L}$ of OA was injected. The QD solution was then transferred to a glovebox as described previously, and were centrifuged at $6000 \mathrm{rpm}$ for $15 \mathrm{~min}$. The supernatant was discarded while the resulting pellet was redispersed in $2 \mathrm{~mL}$ of hexanes. ACA ligand exchange was performed as described previously.

UC sample preparation: For UC measurements, $200 \mu \mathrm{L}$ of a $10 \mathrm{mg} / \mathrm{mL}$ solution of DPA in chlorobenzene were also added to $300 \mu \mathrm{L}$ of NPL/ACA or QD/ACA solutions resulting in a NPL concentration of $2.59 \times 10^{-8} \mathrm{M}$, a QD concentration of $1.51 \times 10^{-6} \mathrm{M}$ (see Supporting Information) $)^{61,62}$, and a DPA concentration of $1.21 \times 10^{-3} \mathrm{M}$.

Nanocrystal characterization: Scanning transmission electron microscopy (STEM) images were recorded with a JEOL JEM-ARM200cF microscope.

Optical characterization: Absorbance spectra were measured by a Shimadzu UV-vis spectrometer (UV-2450, Shimadzu). Steady-state emission was measured with an Ocean Optics 
(HR2000+ES) spectrometer under $405 \mathrm{~nm}$ continuous wave (LDH-D-C-405, PicoQuant) and 532 nm (LDH-P-FA-530L, PicoQuant) pulsed (quasi-steady state, 80MHz) excitation. Time-resolved photoluminescent lifetimes (TRPL) were measured through time-correlated single photon counting (TCSPC) as described in detail previously. ${ }^{55,63}$ The samples were excited by a pulse train $(1 \mathrm{MHz})$ at $532 \mathrm{~nm}$ and photon arrival times were histogrammed by a HydraHarp 400 (PicoQuant). Excess laser scatter was removed by $532 \mathrm{~nm}$ notch filters (ThorLabs), and the UC photoluminescence was detected through an additional $500 \mathrm{~nm}$ short-pass filter, while the NPL or QD emission was detected using a $550 \mathrm{~nm}$ long-pass filter. For $I_{t h}$ measurements, the counts were histogrammed and averaged for $20 \mathrm{~s}$.

Upconversion efficiency: The UCQY $\left(\Phi_{U C}\right)$ was calculated by the following equation with minor modifications, as the QY of the annihilator DPA was not at unity. ${ }^{49}$

$$
\Phi_{U C}=2 \frac{\Phi_{\text {rubrene }}}{\Phi_{D P A}}\left(\frac{A_{\text {rubrene }}}{A_{U C}}\right)\left(\frac{F_{U C}}{F_{\text {rubrene }}}\right)\left(\frac{n_{U C}}{n_{\text {rubrene }}}\right)^{2}
$$

Where $\Phi_{\text {rubrene }}=70 \%, \Phi_{D P A}=73 \%, A, F$, and $n$ represent the QY of rubrene, the QY of 9,10DPA, the absorbance of each solution at $532 \mathrm{~nm}$, integrated fluorescent emission, and refractive indices, respectively of the solutions. In this case, rubrene dissolved in toluene was used as a standard, while the upconverting solution was comprised of NPLs, ACA, and DPA (NPL/ACA/DPA). The absolute QYs of rubrene (in toluene) and DPA (in chlorobenzene) were measured using a Hamamatsu C11347 Quantaurus QY spectrometer while the absorption and emission were measured as described previously. The refractive index of rubrene was estimated to be 1.497 , the refractive index of toluene, the solvent used for the rubrene standard. The refractive index of the NPL/ACA/DPA solution was estimated as 1.434 , calculated using the respective mole fractions and refractive indices of chlorobenzene and hexane used in the solution. The UC efficiencies are determined at a power density of $11 \mathrm{~W} / \mathrm{cm}^{2}$. 


\section{ASSOCIATED CONTENT}

Supporting Information. Experimental methods, upconversion efficiency calculation and supplemental figures: Calculated Stern-Volmer constants and quenching statistics (Table S1); normalized NPL steady-state emission quenching (Figure S1); QD-UC energy level schematic and optical characterization (Figure S2); QD/ACA time resolved and steady-state quenching (Figure S3); NPL emission lifetime post ACA and DPA addition (Figure S4); Ith plot for QD/ACA/DPA (Figure S5); representative UC emission/rubrene emission plot (Figure S6); $I_{t h}$ and UCQY for different QD concentrations (Figure S7); NPL emission intensities at different laser fluences for NPL and NPL/ACA/DPA (Figure S8); NPL emission decay lifetimes for NPL solutions with different amounts of stacking (Figure S9); steady-state emission quenching of a prestacked NPL with ACA (Figure S10); time resolved emission quenching for a highly concentrated NPL with ACA (Figure S11).

\section{AUTHOR INFORMATION}

\section{Corresponding Author}

*E-mail: lnienhaus@fsu.edu

\section{Notes}

The authors declare no competing financial interests.

\section{ACKNOWLEDGEMENT}

We would like to thank Yan Xin for TEM measurements. A portion of this work was performed at the National High Magnetic Field Laboratory, which is supported by the National Science 
Foundation Cooperative Agreement No. DMR-1644779 and the State of Florida. The authors would like to thank the Mattoussi group at Florida State University for aiding in the UV-vis measurements. The authors gratefully acknowledge Florida State University startup funds.

\section{REFERENCES}

(1) Rafique, R.; Baek, S. H.; Park, C. Y.; Chang, S.-J.; Gul, A. R.; Ha, S.; Nguyen, T. P.; Oh, H.; Ham, S.; Arshad, M.; et al. Morphological Evolution of Upconversion Nanoparticles and Their Biomedical Signal Generation. Sci Rep 2018, 8 (1), 1-11.

(2) Hemmer, E.; Acosta-Mora, P.; Méndez-Ramos, J.; Fischer, S. Optical Nanoprobes for Biomedical Applications: Shining a Light on Upconverting and near-Infrared Emitting Nanoparticles for Imaging, Thermal Sensing, and Photodynamic Therapy. J. Mater. Chem. $B$ 2017, 5 (23), 4365-4392.

(3) Zhang, K. Y.; Zhang, T.; Wei, H.; Wu, Q.; Liu, S.; Zhao, Q.; Huang, W. Phosphorescent Iridium(III) Complexes Capable of Imaging and Distinguishing between Exogenous and Endogenous Analytes in Living Cells. Chem. Sci. 2018, 9 (36), 7236-7240.

(4) Shockley, W.; Queisser, H. J. Detailed Balance Limit of Efficiency of P-n Junction Solar Cells. Journal of Applied Physics 1961, 32 (3), 510-519.

(5) Rühle, S. Tabulated Values of the Shockley-Queisser Limit for Single Junction Solar Cells. Solar Energy 2016, 130, 139-147.

(6) Mailoa, J. P.; Akey, A. J.; Simmons, C. B.; Hutchinson, D.; Mathews, J.; Sullivan, J. T.; Recht, D.; Winkler, M. T.; Williams, J. S.; Warrender, J. M.; et al. Room-Temperature SubBand Gap Optoelectronic Response of Hyperdoped Silicon. Nature Communications 2014, 5,3011 .

(7) Ravetz, B. D.; Pun, A. B.; Churchill, E. M.; Congreve, D. N.; Rovis, T.; Campos, L. M. Photoredox Catalysis Using Infrared Light via Triplet Fusion Upconversion. Nature 2019, 565 (7739), 343-346.

(8) Zhou, J.; Liu, Q.; Feng, W.; Sun, Y.; Li, F. Upconversion Luminescent Materials: Advances and Applications. Chem. Rev. 2015, 115 (1), 395-465.

(9) Majek, M.; Faltermeier, U.; Dick, B.; Pérez-Ruiz, R.; Jacobi von Wangelin, A. Application of Visible-to-Uv Photon Upconversion to Photoredox Catalysis: The Activation of Aryl Bromides. Chem. Eur. J. 2015, 21 (44), 15496-15501.

(10) Häring, M.; Pérez-Ruiz, R.; Wangelin, A. J. von; Díaz, D. D. Intragel Photoreduction of Aryl Halides by Green-to-Blue Upconversion under Aerobic Conditions. Chem. Commun. 2015, 51 (94), 16848-16851.

(11) Shan, B.; Li, T.-T.; Brennaman, M. K.; Nayak, A.; Wu, L.; Meyer, T. J. Charge Transfer from Upconverting Nanocrystals to Semiconducting Electrodes: Optimizing Thermodynamic Outputs by Electronic Energy Transfer. J. Am. Chem. Soc. 2019, 141 (1), 463-471.

(12) Marcus, R. A. On the Theory of Oxidation-Reduction Reactions Involving Electron Transfer. I. J. Chem. Phys. 1956, 24 (5), 966-978. 
(13) Dexter, D. L. A Theory of Sensitized Luminescence in Solids. J. Chem. Phys. 1953, 21, 836-850.

(14) Haefele, A.; Blumhoff, J.; Khnayzer, R. S.; Castellano, F. N. Getting to the (Square) Root of the Problem: How to Make Noncoherent Pumped Upconversion Linear. The Journal of Physical Chemistry Letters 2012, 3 (3), 299-303.

(15) Schmidt, T. W.; Castellano, F. N. Photochemical Upconversion: The Primacy of Kinetics. The Journal of Physical Chemistry Letters 2014, 5 (22), 4062-4072.

(16) Amemori, S.; Sasaki, Y.; Yanai, N.; Kimizuka, N. Near-Infrared-to-Visible Photon Upconversion Sensitized by a Metal Complex with Spin-Forbidden yet Strong S 0 -T 1 Absorption. Journal of the American Chemical Society 2016, 138 (28), 8702-8705.

(17) Islangulov, R. R.; Kozlov, D. V.; Castellano, F. N. Low Power Upconversion Using MLCT Sensitizers. Chem. Commun. 2005, No. 30, 3776-3778.

(18) Monguzzi, A.; Mezyk, J.; Scotognella, F.; Tubino, R.; Meinardi, F. Upconversion-Induced Fluorescence in Multicomponent Systems: Steady-State Excitation Power Threshold. Phys. Rev. B 2008, 78 (19), 195112.

(19) Cheng, Y. Y.; Khoury, T.; Clady, R. G. C. R.; Tayebjee, M. J. Y.; Ekins-Daukes, N. J.; Crossley, M. J.; Schmidt, T. W. On the Efficiency Limit of Triplet-Triplet Annihilation for Photochemical Upconversion. Phys. Chem. Chem. Phys. 2010, 12 (1), 66-71.

(20) Singh-Rachford, T. N.; Castellano, F. N. Photon Upconversion Based on Sensitized TripletTriplet Annihilation. Coord. Chem. Rev. 2010, 254 (21-22), 2560-2573.

(21) Singh-Rachford, T. N.; Castellano, F. N. Triplet Sensitized Red-to-Blue Photon Upconversion. J. Phys. Chem. Lett. 2010, 1 (1), 195-200.

(22) Joarder, B.; Yanai, N.; Kimizuka, N. Solid-State Photon Upconversion Materials: Structural Integrity and Triplet-Singlet Dual Energy Migration. J. Phys. Chem. Lett. 2018, 9 (16), 4613-4624.

(23) Dzebo, D.; Moth-Poulsen, K.; Albinsson, B. Robust Triplet-Triplet Annihilation Photon Upconversion by Efficient Oxygen Scavenging. Photochem. Photobiol. Sci. 2017, 16 (8), 1327-1334.

(24) Kim, J.-H.; Deng, F.; Castellano, F. N.; Kim, J.-H. High Efficiency Low-Power Upconverting Soft Materials. Chem. Mater. 2012, 24 (12), 2250-2252.

(25) Nienhaus, L.; Wu, M.; Bulovic, V.; Baldo, M. A.; Bawendi, M. G. Using Lead Chalcogenide Nanocrystals as Spin Mixers: A Perspective on near-Infrared-to-Visible Upconversion. Dalton Trans. 2018, 47, 8509-8516.

(26) Kim, J.; Wong, C. Y.; Scholes, G. D. Exciton Fine Structure and Spin Relaxation in Semiconductor Colloidal Quantum Dots. Acc. Chem. Res. 2009, 42 (8), 1037-1046.

(27) You, Z.-Q.; Hsu, C.-P. Theory and Calculation for the Electronic Coupling in Excitation Energy Transfer. Int. J. Quantum Chem. 2014, 114 (2), 102-115.

(28) Scholes, G. D.; Rumbles, G. Excitons in Nanoscale Systems. Nat Mater 2006, 5 (9), 683696.

(29) De Roo, J.; Huang, Z.; Schuster, N. J.; Hamachi, L. S.; Congreve, D. N.; Xu, Z.; Xia, P.; Fishman, D. A.; Lian, T.; Owen, J. S.; et al. Anthracene Diphosphate Ligands for CdSe Quantum Dots; Molecular Design for Efficient Upconversion. Chem. Mater. 2020, 32 (4), 1461-1466.

(30) Okumura, K.; Mase, K.; Yanai, N.; Kimizuka, N. Employing Core-Shell Quantum Dots as Triplet Sensitizers for Photon Upconversion. Chem. Eur. J. 2016, 22 (23), 7721-7726. 
(31) Mahboub, M.; Huang, Z.; Tang, M. L. Efficient Infrared-to-Visible Upconversion with Subsolar Irradiance. Nano Lett. 2016, 16 (11), 7169-7175.

(32) Huang, Z.; Li, X.; Mahboub, M.; Hanson, K. M.; Nichols, V. M.; Le, H.; Tang, M. L.; Bardeen, C. J. Hybrid Molecule-Nanocrystal Photon Upconversion across the Visible and near-Infrared. Nano Lett. 2015, 15 (8), 5552-5557.

(33) Huang, Z.; Tang, M. L. Designing Transmitter Ligands That Mediate Energy Transfer between Semiconductor Nanocrystals and Molecules. J. Am. Chem. Soc. 2017, 139 (28), 9412-9418.

(34) Ithurria, S.; Dubertret, B. Quasi 2D Colloidal CdSe Platelets with Thicknesses Controlled at the Atomic Level. J. Am. Chem. Soc. 2008, 130 (49), 16504-16505.

(35) Ithurria, S.; Tessier, M. D.; Mahler, B.; Lobo, R. P. S. M.; Dubertret, B.; Efros, A. L. Colloidal Nanoplatelets with Two-Dimensional Electronic Structure. Nature Materials 2011, 10 (12), 936-941.

(36) Abécassis, B.; Tessier, M. D.; Davidson, P.; Dubertret, B. Self-Assembly of CdSe Nanoplatelets into Giant Micrometer-Scale Needles Emitting Polarized Light. Nano Lett. 2014, 14 (2), 710-715.

(37) Gao, Y.; Weidman, M. C.; Tisdale, W. A. CdSe Nanoplatelet Films with Controlled Orientation of Their Transition Dipole Moment. Nano Lett. 2017, 17 (6), 3837-3843.

(38) Singh, S.; Tomar, R.; ten Brinck, S.; De Roo, J.; Geiregat, P.; Martins, J. C.; Infante, I.; Hens, Z. Colloidal CdSe Nanoplatelets, A Model for Surface Chemistry/Optoelectronic Property Relations in Semiconductor Nanocrystals. J. Am. Chem. Soc. 2018, 140 (41), 13292-13300.

(39) Spittel, D.; Poppe, J.; Meerbach, C.; Ziegler, C.; Hickey, S. G.; Eychmüller, A. Absolute Energy Level Positions in CdSe Nanostructures from Potential-Modulated Absorption Spectroscopy (EMAS). ACS Nano 2017, 11 (12), 12174-12184.

(40) Kudlacik, D.; Sapega, V. F.; Yakovlev, D. R.; Kalitukha, I. V.; Shornikova, E. V.; Rodina, A. V.; Ivchenko, E. L.; Dimitriev, G. S.; Nasilowski, M.; Dubertret, B.; et al. Single and Double Electron Spin-Flip Raman Scattering in CdSe Colloidal Nanoplatelets. Nano Lett. 2020, 20 (1), 517-525.

(41) Diroll, B. T.; Chen, M.; Coropceanu, I.; Williams, K. R.; Talapin, D. V.; Guyot-Sionnest, P.; Schaller, R. D. Polarized Near-Infrared Intersubband Absorptions in CdSe Colloidal Quantum Wells. Nature Communications 2019, 10 (1), 1-9.

(42) Morgan, D. P.; Kelley, D. F. Exciton Localization and Radiative Lifetimes in CdSe Nanoplatelets. J. Phys. Chem. C 2019, 123 (30), 18665-18675.

(43) Pedetti, S.; Ithurria, S.; Heuclin, H.; Patriarche, G.; Dubertret, B. Type-II CdSe/CdTe Core/Crown Semiconductor Nanoplatelets. J. Am. Chem. Soc. 2014, 136 (46), 1643016438.

(44) Nasilowski, M.; Mahler, B.; Lhuillier, E.; Ithurria, S.; Dubertret, B. Two-Dimensional Colloidal Nanocrystals. Chem. Rev. 2016, 116 (18), 10934-10982.

(45) Antanovich, A.; Prudnikau, A.; Matsukovich, A.; Achtstein, A.; Artemyev, M. SelfAssembly of CdSe Nanoplatelets into Stacks of Controlled Size Induced by Ligand Exchange. J. Phys. Chem. C 2016, 120 (10), 5764-5775.

(46) Zhou, Y.; Buhro, W. E. Reversible Exchange of L-Type and Bound-Ion-Pair X-Type Ligation on Cadmium Selenide Quantum Belts. J. Am. Chem. Soc. 2017, 139 (37), 1288712890. 
(47) Mahboub, M.; Maghsoudiganjeh, H.; Pham, A. M.; Huang, Z.; Tang, M. L. Triplet Energy Transfer from $\mathrm{PbS}(\mathrm{Se})$ Nanocrystals to Rubrene: The Relationship between the Upconversion Quantum Yield and Size. Advanced Functional Materials 2016, 26 (33), 6091-6097.

(48) Radiunas, E.; Raišys, S.; Jursenas, S.; Jozeliūnaitė, A.; Jarovskis, T.; Šinkevičiūtė, U.; Orentas, E.; Kazlauskas, K. Understanding the Limitations of NIR-to-Visible Photon Upconversion in Phthalocyanine-Sensitized Rubrene Systems. J. Mater. Chem. C 2020.

(49) Singh-Rachford, T. N.; Castellano, F. N. Photon Upconversion Based on Sensitized TripletTriplet Annihilation. Coord. Chem. Rev. 2010, 254 (21-22), 2560-2573.

(50) Shornikova, E. V.; Biadala, L.; Yakovlev, D. R.; Sapega, V. F.; Kusrayev, Y. G.; Mitioglu, A. A.; Ballottin, M. V.; Christianen, P. C. M.; Belykh, V. V.; Kochiev, M. V.; et al. Addressing the Exciton Fine Structure in Colloidal Nanocrystals: The Case of CdSe Nanoplatelets. Nanoscale 2018, 10 (2), 646-656.

(51) Tabernig, S. W.; Daiber, B.; Wang, T.; Ehrler, B. Enhancing Silicon Solar Cells with Singlet Fission: The Case for Förster Resonant Energy Transfer Using a Quantum Dot Intermediate. JPE 2018, 8 (2), 022008.

(52) Guzelturk, B.; Erdem, O.; Olutas, M.; Kelestemur, Y.; Demir, H. V. Stacking in Colloidal Nanoplatelets: Tuning Excitonic Properties. ACS Nano 2014, 8 (12), 12524-12533.

(53) Guzelturk, B.; Demir, H. V. Near-Field Energy Transfer Using Nanoemitters For Optoelectronics. Advanced Functional Materials 2016, 26 (45), 8158-8177.

(54) Wieghold, S.; Bieber, A. S.; VanOrman, Z. A.; Daley, L.; Leger, M.; Correa-Baena, J.-P.; Nienhaus, L. Triplet Sensitization by Lead Halide Perovskite Thin Films for Efficient SolidState Photon Upconversion at Subsolar Fluxes. Matter 2019, 1 (3), 705-719.

(55) Wieghold, S.; Nienhaus, L. Precharging Photon Upconversion: Interfacial Interactions in Solution-Processed Perovskite Upconversion Devices. J. Phys. Chem. Lett. 2020.

(56) Cruz, C. D.; Choi, H. H.; Podzorov, V.; Chronister, E. L.; Bardeen, C. J. Photon Upconversion in Crystalline Rubrene: Resonant Enhancement by an Interband State. $J$. Phys. Chem. C 2018, 122 (31), 17632-17642.

(57) Bertrand, G. H. V.; Polovitsyn, A.; Christodoulou, S.; Khan, A. H.; Moreels, I. Shape Control of Zincblende CdSe Nanoplatelets. Chem. Commun. 2016, 52 (80), 11975-11978.

(58) Bertrand, G. H. V.; Polovitsyn, A.; Christodoulou, S.; Khan, A. H.; Moreels, I. Shape Control of Zincblende CdSe Nanoplatelets. Chem. Commun. 2016, 52 (80), 11975-11978.

(59) Ithurria, S.; Tessier, M. D.; Mahler, B.; Lobo, R. P. S. M.; Dubertret, B.; Efros, Al. L. Colloidal Nanoplatelets with Two-Dimensional Electronic Structure. Nature Materials 2011, 10, 936.

(60) Yeltik, A.; Delikanli, S.; Olutas, M.; Kelestemur, Y.; Guzelturk, B.; Demir, H. V. Experimental Determination of the Absorption Cross-Section and Molar Extinction Coefficient of Colloidal CdSe Nanoplatelets. J. Phys. Chem. C 2015, 119 (47), 2676826775.

(61) Yu, W. W.; Qu, L.; Guo, W.; Peng, X. Experimental Determination of the Extinction Coefficient of CdTe, CdSe, and CdS Nanocrystals. Chem. Mater. 2003, 15 (14), 2854-2860.

(62) Wieghold, S.; Bieber, A. S.; VanOrman, Z. A.; Nienhaus, L. Influence of Triplet Diffusion on Lead Halide Perovskite-Sensitized Solid-State Upconversion. J. Phys. Chem. Lett. 2019, 3806-3811. 